\title{
Password Based Key Exchange Protocols on Elliptic Curves Which Conceal the Public Parameters
}

\author{
Julien Bringer ${ }^{1}$, Hervé Chabanne ${ }^{1,2}$, and Thomas Icart ${ }^{3}$ \\ 1 Sagem Sécurité \\ 2 Télécom ParisTech \\ 3 This work has been done while this author was affiliated with \\ Sagem Sécurité and the University of Luxembourg
}

\begin{abstract}
We here describe a new Password-based Authenticated Key Exchange (PAKE) protocol based on elliptic curve cryptography. We prove it secure in the Bellare-Pointcheval-Rogaway (BPR) model. A significant novelty in our work is that the elliptic curve public parameters remain private. This is important in the context of ID contactless devices as, in this case, there will exist most probably a way to link these parameters with the nationality of the ID document owners.
\end{abstract}

Keywords: Password-based Authenticated Key Exchange, Elliptic Curves, Privacy.

\section{Introduction}

To enable secure communication over insecure channels, two parties encrypt and authenticate their messages using a shared secret key, usually obtained through key exchange. A key exchange protocol enables the two parties to establish a common secret in an authenticated way (Authenticated Key Exchange, AKE). The goal is for the session key to be known only by the parties involved in the protocol; the session key should be indistinguishable from a random data. Password-based key exchange protocols are a convenient way to achieve this. The two parties rely on a shared low-entropy secret (e.g. a four-digit PIN) to derive a common high-entropy session key.

Password-Based Authenticated Key Exchange (PAKE) protocols are today considered in the context of identity documents to ensure the security of the communications between the chip and a reader [18. With machine readable travel documents (MRTD, cf. International Civil Aviation Organization specifications [15]), the data stored on the machine readable zone (MRZ) are seen as low-entropy shared information between the reader and the chip to establish a secure link. For efficiency constraints, the protocols usually rely on elliptic curves. Moreover, when these protocols will be used in real ID documents, the parameters will almost surely depend on the nationality of the document owner (each country keeps usually its freedom of choice in matter of cryptographic parameters with respect to its own evaluation of the associated level of security). 
However, if one eavesdrops the communication resulting from executions of an AKE protocol based on elliptic curves, then he would learn some elliptic curve points and would be able to obtain the elliptic curve parameters 2. He therefore retrieves the owner's nationality as a side information, thus leading to a privacy leakage and security issues. This is our motivation for finding PAKE protocols based on elliptic curves while hiding the elliptic curve parameters. To the best of our knowledge, our work is the first to provide a secure solution to this problem.

\subsection{Related Works}

AKE protocols. Password-based authenticated key exchange was considered first by Bellovin and Merritt [5]. The goal is to authenticate a key exchange between two parties based on simple passwords possibly from a small dictionary that an adversary may know. The basic idea behind the different schemes described in [5] was to encrypt some messages of the key exchange (hence the acronym EKE for Encrypted Key Exchange). This work was followed by many variants and later on several security analysis in different models, e.g. [5, 6, 3, 4, 9, 10, 8. The main issue is the resistance against offline dictionary attacks.

One of the most well-known variant of EKE is Diffie-Hellman EKE (DH-EKE) which is merely a DH key exchange where at least one message exchanged under DH is encrypted via the password. Bellare et al. introduced in [3] a formal security model to grasp the specificity of password-based key exchange. This model was used later in 9, 10 to establish the security of DH-EKE under ideal assumptions (namely the ideal cipher model and the Random Oracle Model, ROM). However ideal cipher model is not easily applicable to elliptic curves and thus in its classical version, DH-EKE is not well adapted to elliptic curves. A naive application of the encryption step of $\mathrm{DH}-\mathrm{EKE}$ to elliptic curves points would lead to an insecure scheme. Indeed, due to the redundancy in a point representation, partition attacks [16] are made possible to distinguish possible passwords from impossible ones and the security against offline dictionary attacks does not hold (the main idea is that a decryption with a bad password of an encrypted point would most probably not be a point over the elliptic curve). In [8, a modification of the scheme is suggested by using either a point of the curve or a point over its twist. Although, this enables to withstand the security issue, the scheme is not proved in the model of [3] and becomes much less simple than DH-EKE.

Another well-known and widely used PAKE is SPEKE (Simple Password Exponential Key Exchange) [14. It is part of the IEEE P1363.2 standard. Likewise, it is based on Diffie-Hellman key exchange but the password is here used to select the generator of the DH key exchange, which is then operated without encrypted messages. To do so, a hash of the password is used to generate a group element, and the security is based on the ROM. Here again security against offline dictionary attacks does not hold if you do not have a way to hash into elliptic curves (cf. also Remark 5). 
Finally, in both cases, DH-EKE and SPEKE do not hide the curve parameters as they can easily be deduced from 2 eavesdropped points.

The BPR (Bellare-Pointcheval-Rogaway) model. Several security models have been suggested to analyze the security of password-based AKE. The BPR model [3] is now considered as a standard model for PAKE protocols. It captures well the security requirements that a PAKE should satisfy. In particular even if protocols remain subject to online guessing attacks, they should thwart offline dictionary attacks. Different protocols have been shown secure in this model. The model is based on the Find-Then-Guess principle where an adversary - mounting an active attack against several protocol instances running concurrently - should not be able to determine whether a session key is the actual one, i.e. that the key should be indistinguishable from a random string. This also ensures an implicit authentication between the two parties involved in the protocol. The model is refined in [1] by requiring that all the concurrent session keys look random (RealOr-Random principle).

Admissible Encodings. The notion of admissible encoding has been introduced by Boneh-Franklin in order to hash into elliptic curves since it is required for their Identity-Based Encryption scheme [7]. Later, Coron and Icart [12] have introduced a more general notion of admissible encodings. These encodings are built out of deterministic functions that map bit strings into elliptic curves such the ones of [13, 17].

An admissible encoding from $\{0,1\}^{*}$ into a group is a function that enables to transform any bit string into a group element. Moreover, to comply with the definition, there must exist a polynomial-time inversion algorithm that can compute a bit string from a group element. The existence of this algorithm ensures that a random group element is mapped into a random bit string. Thanks to these encodings, 12 describes a way to create a random oracle into an elliptic curve. From [12, we here only use the fact that a random point can be represented by a random bit string.

This property can be used within the DH-EKE protocol, as it is much safer to encrypt random bit strings, especially when the key is directly derived from a low entropy password.

\subsection{Our Contribution}

We provide the first PAKE protocol which ensures that the elliptic curve public parameters remain hidden. This holds even against dictionary attacks. This is made possible thanks to the existence of admissible encodings. Since each elliptic curve point can be seen as a random bit string, it enables to encrypt a point by processing a bit string through a block cipher. The password can here be seen as a seed for computing the secret key used in the block cipher. This has a direct application within DH-EKE, as an eavesdropper cannot verify which elliptic curve parameters are used. Since eavesdroppers only see "random" bit 
strings, whatever are the elliptic curve public parameters, they cannot tell which ones are used.

This first construction is a direct application of admissible encodings on elliptic curves. Moreover, we provide a second PAKE protocol where we exploit the fact that the knowledge of the elliptic curve parameters can be interpreted as a shared secret. This is our main result as this enables to drop out the encryption of EKE while the protocol can still be proved secure. We introduce complexity assumptions based on the discrete logarithm problem ensuring that finding one bit string which represents 2 points with known discrete logarithm from 2 different curves is hard. These assumptions enable us to prove the security of our Diffie-Hellman AKE protocol where the password directly relies on the elliptic curve parameters. The hardness of these new complexity assumptions holds in the generic group model (as it is proved in the extended version of the paper [11]).

\section{Definitions}

\subsection{Admissible Encoding and Admissible Representation}

We here recall the definition of an admissible encoding.

Definition 1. Given 2 random variables $X$ and $Y$ over a set $S$, we say that the distribution of $X$ and $Y$ are $(\epsilon)$-statistically indistinguishable if:

$$
\sum_{s \in S}|\operatorname{Pr}(X=s)-\operatorname{Pr}(Y=s)|<\epsilon
$$

Moreover, given a security parameter, two distributions are statistically indistinguishable if they are $(\epsilon)$-statistically indistinguishable for an $\epsilon$ negligible in the security parameter.

Definition 2 (Admissible Encoding, [12]). A function $F: S \rightarrow R$ is said to be an $\varepsilon$-admissible encoding if:

1. F is computable in deterministic polynomial time;

2. There exists such a probabilistic polynomial time algorithm $\mathcal{I}_{F}$ : given $r \in R$ as input, $\mathcal{I}_{F}$ outputs $s$ such that either $F(s)=r$ or $s=\perp$, and the distribution of $s$ is $\varepsilon$-statistically indistinguishable from the uniform distribution in $S$ when $r$ is uniformly distributed in $R$.

When an admissible encoding from $\{0,1\}^{L}$ into a curve exists, its inversion algorithm $\mathcal{I}_{F}$ enables to transform uniformly distributed elliptic curve points into uniformly distributed bit strings. Encrypting an elliptic curve point thus becomes easier when it is represented as a bit string. In particular, no trivial partition attack is possible. 
Icart's Mapping in Characteristic 2. The equation which defines an elliptic curve $E_{a, b}$ in characteristic 2 is of general form:

$$
\left(E_{a, b}\right) \quad Y^{2}+X Y=X^{3}+a X^{2}+b
$$

where $a$ and $b$ are elements of $\mathbb{F}_{2^{n}}$. For an odd $n$, the map $x \mapsto x^{3}$ is a bijection. Let

$$
\begin{aligned}
f_{a, b}: \mathbb{F}_{2^{n}} & \mapsto\left(\mathbb{F}_{2^{n}}\right)^{2} \\
u & \mapsto\left(x, u x+v^{2}\right)
\end{aligned}
$$

where $v=a+u+u^{2}$ and $x=\left(v^{4}+v^{3}+b\right)^{1 / 3}+v$. It is clear that, whenever computing a cube root is an exponentiation, computing $f_{a, b}$ is a deterministic polynomial time algorithm.

Lemma 1 (Hashing into $E_{a, b},[\mathbf{1 3}]$ ). Let $\mathbb{F}_{2^{n}}$ be a field with $n$ odd. For any $u \in \mathbb{F}_{2^{n}}, f_{a, b}(u)$ is a point of $E_{a, b}$.

We here focus on characteristic 2 version of [13] for two reasons: the computation is simpler than in the general case, and the inverting algorithm is deterministic and easy to implement [11. Note that this encoding is not the only known general encoding for elliptic curves. In [17, Shallue and van de Woestijne proposed another encoding. We choose to introduce only the encoding from [13] as the encoding from [17] is not as simple to describe. But it is possible to adapt our work to any existing admissible encoding.

Let $E$ be an elliptic curve over a field $\mathbb{F}_{2^{n}}$. From such a point encoding $f$ and a generator $G$ of the group of points, an admissible encoding $F$ from $\{0,1\}^{2 n}$ to $E$ can be constructed. Let $l$ be a $2 n$-bit long string. This string is split in 2 substrings $u \| \lambda$ where $\lambda$ is seen as a $n$-bit integer. We then introduce what we call in the sequel the Coron-Icart admissible encoding:

$$
F(l)=f(u)+\lambda \cdot G
$$

The resulting function is proved to be an admissible encoding in [12] with a negligible $\epsilon$. The main condition on $f$ is to be an encoding that satisfies a condition weaker than in Definition 2, where the inversion algorithm needs to work only on a polynomial fraction of the inputs and where the statistically indistinguishability is measured only with respect to this fraction. Such encoding is denoted in 12 a weak encoding.

Admissible Representation. We introduce below the notion of admissible representations. These representations are the outputs of $\mathcal{I}_{F}$, when it is applied to an elliptic curve point.

Definition 3 (Admissible Representation). Assume that $F$ is an admissible encoding from $S$ to $R$. For any $r \in R$, we define as an admissible representation of $r$ any output of $\mathcal{I}_{F}(r)$. 
An element $r \in R$ may have many different admissible representations. Furthermore, an uniformly random $r \in R$ has an uniformly random admissible representation $s \in S$. For instance, following Eq. (11) a random point $P$ of an elliptic curve admits an admissible representation of the form $(u, \lambda)$ where $u \| \lambda$ is a random bit string of size $2 n$.

\subsection{BPR Security Model}

This model defines the notions of partnership, session key freshness and security against dictionary attacks. The model considers a set of honest players who do not deviate from the protocol. The adversary controls all the communications network. This is an active adversary formalized through queries. Users can have many protocol instances running concurrently. The adversary can create, modify, or forward messages and has oracle access to the user instances.

Let $A$ and $B$ be two users which can be part of the key exchange protocol $P$. Several concurrent instances may run in different executions of $P$ : they are denoted by $A_{i}$ and $B_{j}$. The server and the user share a low-entropy secret $p w$ uniformly drawn from a dictionary of size $N$.

Oracles. The protocol $P$ consists of the execution of a key exchange algorithm. It is an interactive protocol between $A_{i}$ and $B_{j}$ that provides the instances of $A$ and $B$ with a session key $s k$. The adversary $\mathcal{A}$ has access to the following oracles for controlling the interactions.

- Execute $\left(A_{i}, B_{j}\right)$ simulates a passive attack where $\mathcal{A}$ eavesdrops the communication. It causes an honest execution of $P$ between fresh instances $A_{i}$ and $B_{j}$.

- $\operatorname{Send}\left(U_{i}, m\right)$ models $\mathcal{A}$ sending a message $m$ to instance $U_{i}(U=A$ or $B)$. The output is the message generated by $U$ in processing the message $m$ according to the protocol and the state of the instance. It simulates an active attack.

- REveaL $\left(U_{i}\right)$ returns the session key of the input instance. This query models the misuse of the session key by instance $U_{i}$. The query is only available to the adversary if the targeted instance actually holds a session key (i.e. if the protocol has correctly terminated).

Security Notions. Two instances are defined as partnered if both instances have terminated correctly with the same session key. The freshness notion captures the fact that a session key has not been directly leaked. An instance is said to be fresh in the current protocol execution if the instance has terminated and neither a REVEAL query has been called on the instance nor on a partnered instance.

The $\operatorname{Test}\left(U_{i}\right)$ query models the semantic security of the session key. It is available to the adversary only if the aimed instance is fresh. When called, the oracle tosses a coin $b$ and returns the session key $s k$ if $b=0$ or a random value (from the domain of keys) if $b=1$. 
The AKE security is then defined as follows. By controlling executions of the protocol $P$, the adversary $\mathcal{A}$ tries to learn information on the session keys. The game is initialized by drawing a password $p w$ from the dictionary and by letting $\mathcal{A}$ ask a polynomial number of queries. At the end of the game, $\mathcal{A}$ outputs its guess $b^{\prime}$ for the bit output by the TEST oracle.

The AKE advantage of the adversary $\mathcal{A}$ for the key exchange protocol $P$ is denoted

$$
\operatorname{Adv}_{P}^{\text {ake }}(\mathcal{A})=\left|\operatorname{Pr}\left[b^{\prime}=b\right]-\frac{1}{2}\right|
$$

Definition 4. The protocol $P$ is said to be AKE-secure if the adversary's advantage is negligible in the security parameter, for any polynomially bounded adversary.

A strategy of proof consists generally in the simulation of all the oracles to show that there is no leakage.

Remark 1. An oracle CORRUPT is also available in this model to analyze the forward secrecy. When called with respect to one player, the adversary will obtain the player's password. For AKE with forward secrecy, the TEST query should not be related to a player corrupted before the TEST query. Nevertheless, corruption after the query is allowed.

\subsection{Classical Assumption}

We recall the classical Computational Diffie-Hellman (CDH) assumption.

Definition 5 (CDH Assumption). Let $E$ be an elliptic curve and $G$ be a generator of a subgroup of points of prime order. Let $\mathcal{A}$ be an algorithm that:

- inputs two random points $P=a \cdot G$ and $Q=b \cdot G$;

- and outputs $R=a b \cdot G$.

The CDH assumption ensures that the best polynomial time adversary has a negligible probability of success, when the probability is taken over $P$ and $Q$.

Throughout this work, we define $\mathrm{CDH}_{G}(P, Q)$ to be the correct value of $R$. We introduce in the next section assumptions related to this problem when the elliptic curve parameters are unknown. In particular, given two elliptic curves $E_{1}, E_{2}$, we rely on the hardness of finding two admissible representations $l$ and $l^{\prime}$ such that the points $\operatorname{CDH}\left(F_{1}(l), F_{1}\left(l^{\prime}\right)\right) \in E_{1}$ and $\operatorname{CDH}\left(F_{2}(l), F_{2}\left(l^{\prime}\right)\right) \in E_{2}$ are known (with $F_{i}$ an admissible encoding into $E_{i}, i \in\{1,2\}$ ).

\section{A New Family of Complexity Assumptions}

In order to prove the strength of our protocol, we introduce complexity assumptions that arise from the fact that we are using in the same scheme different elliptic curve parameters. As already mentioned, these assumptions are new due 
to our specific setting. However, we strongly justify the difficulty of these assumptions in the sequel.

Throughout this section, we use the following definitions. Let $k$ be a security parameter. Let $S$ be a set of $N=\operatorname{poly}(k)$ sets of elliptic curve parameters: $\left\{a_{i}, b_{i}, q_{i}, G_{i}\right\}_{i \in[1, N]}$ over a field $\mathbb{F}_{2^{n}}$ (i.e. elliptic curves $E_{i}:=E_{a_{i}, b_{i}}$ over $\mathbb{F}_{2^{n}}$ with a point $G_{i}$, generator of a subgroup of points of order $q_{i}$ ) such that:

- for each $i$, an admissible encoding (cf. Definition 2) exists over $E_{a_{i}, b_{i}}$;

$-q_{i}$ is a prime integer and its cofactor is 2 (more generally we need the same cofactor for all curves);

- for all $i \neq j$, we have $q_{i} \neq q_{j}$.

The last point ensures that there does not exist an isomorphism between the different curves. It is important since it ensures that the discrete logarithm of a point over $E_{i}$ is not related to a discrete logarithm over another $E_{j}$.

Let $F_{i}$ be the admissible encoding associated to $E_{i}$. In the sequel, we mainly focus on the Coron-Icart admissible encoding obtained via Equation. (11) (Section 2.1) with the point encoding from [13]. It ensures that an admissible representation of size $2 n$ exists for almost all points.

\subsection{Hard Problems Around the Discrete Logarithm of the Points $P_{i}$}

One question arises from this setting: Given a bit string $l$, is the discrete logarithm of each $P_{i}=F_{i}(l)$ in basis $G_{i}$ still hard to compute?

Since an admissible encoding has an inversion algorithm, over each curve $E_{i}$, given a point with an unknown discrete logarithm, we can almost always (except with a negligible probability) compute one of its admissible representations and thus we have:

Lemma 2. Assume that $F_{i}$ is an admissible encoding. Computing the discrete logarithm of any $P_{i}=F_{i}(l)$ with the knowledge of $l$ is as hard as solving the discrete logarithm problem over the curve $E_{i}$.

When an adversary computes an admissible representation $l$ of a point $P_{i}$ over $E_{i}$, we also want that for each admissible representation, his advantage on the discrete logarithm of $P_{j}=F_{j}(l)$ in basis $G_{j}$ over $E_{j}$, for some $j \neq i$, remains negligible.

\section{Definition 6 (Admissible Encoding Twin Discrete Logarithm Assump-} tion). Let $\mathcal{A}$ be an algorithm that:

- inputs $S$;

- outputs $l$ and a pair $\left(r_{i}, r_{j}\right) \in\left(\mathbb{Z} / q_{i} \mathbb{Z}^{\times} \times \mathbb{Z} / q_{j} \mathbb{Z}^{\times}\right)$such that $P_{i}=F_{i}(l)=r_{i} \cdot G_{i}$ and $P_{j}=F_{j}(l)=r_{j} \cdot G_{j}$.

The AET-DL assumption holds if any polynomial algorithm succeeds with a negligible probability, when the probability is taken over $S$. 
Remark 2. This assumption can be expressed differently for the Coron-Icart admissible encoding of Eq. (1). Indeed, for this encoding, $l$ is a pair of values $(u, \lambda)$ such that $F_{i}(l)=f_{i}(u)+\lambda \cdot G_{i}$. Clearly, finding $u$ and a pair $\left(r_{i}, r_{j}\right)$ such that $f_{i}(u)=r_{i} \cdot G_{i}$ and $f_{j}(u)=r_{j} \cdot G_{j}$ is equivalent to solving the AET-DL problem. The problem is thus to find $r_{1}, r_{2}$ such that $f_{1}^{-1}\left(r_{1} \cdot G_{1}\right) \cap f_{2}^{-1}\left(r_{2} \cdot G_{2}\right)$ is a non-empty set. For a random pair $\left(G, G^{\prime}\right) \in E_{i} \times E_{j}$ the probability to have a $u$ such that $f_{i}(u)=G$ and $f_{j}(u)=G^{\prime}$ is at most $4 \times \frac{4}{2^{n}}=2^{-(n-4)}$ for the Icart mapping, because any point has at most 4 preimages (see [13]) through this mapping. Since the scalar multiplication is a one-way map in each $E_{i}$, it is computationally hard to find such pairs.

In addition to the above justification of the difficulty of the AET-DL problem, we formally prove in the generic group model the hardness of this AET-DL problem in 11 .

\section{Definition 7 (Admissible Encoding Twin Computational Diffie-} Hellman Assumption). Let $\mathcal{A}$ be an algorithm that:

- inputs $S$ and $l$;

- outputs $l^{\prime}$ and a pair of points $\left(R_{i}, R_{j}\right)$ (both points different from the neutral element) such that

$\mathrm{CDH}_{G_{i}}\left(F_{i}(l), F_{i}\left(l^{\prime}\right)\right)=R_{i}$ and $\mathrm{CDH}_{G_{j}}\left(F_{j}(l), F_{j}\left(l^{\prime}\right)\right)=R_{j}$.

The AET-CDH assumption holds if any polynomial time adversary has a negligible probability of success, when the probability is taken over $S$ and $l$.

This assumption is stronger than the AET-DL assumption because the AET$\mathrm{CDH}$ problem can be solved using the $l, r_{i}, r_{j}$ of the AET-DL assumption.

Remark 3. Due to the special form of admissible encodings defined by Eq. (1), $F_{i}$ can be replaced by $f_{i}$ in this assumption. For this reason, the AET-CDH assumption ensures that an adversary, who receives a bit string $u$, cannot compute $u^{\prime}$ such that over $E_{i}$ and $E_{j}$ he knows both $\operatorname{CDH}_{G_{i}}\left(f_{i}\left(u^{\prime}\right), f_{i}(u)\right)$ and $\mathrm{CDH}_{G_{j}}\left(f_{j}\left(u^{\prime}\right), f_{j}(u)\right)$. It is easily seen that $\mathrm{CDH}_{G_{i}}\left(f_{i}\left(u^{\prime}\right), f_{i}(u)\right)=R_{i}$ implies $\mathrm{CDH}_{f_{i}(u)}\left(G_{i}, R_{i}\right)=f_{i}\left(u^{\prime}\right)$. The AET-CDH problem is thus to find $R_{i} \in E_{i}$ and $R_{j} \in E_{j}$ such that

$$
f_{i}^{-1}\left(\mathrm{CDH}_{f_{i}(u)}\left(G_{i}, R_{i}\right)\right) \cap f_{j}^{-1}\left(\mathrm{CDH}_{f_{j}(u)}\left(G_{j}, R_{j}\right)\right) \neq \emptyset
$$

As above, the probability for a random pair $\left(G, G^{\prime}\right) \in E_{i} \times E_{j}$ to have a $u^{\prime}$ such that $f_{i}\left(u^{\prime}\right)=G$ and $f_{j}\left(u^{\prime}\right)=G^{\prime}$ is at most $4 \times \frac{4}{2^{n}}=2^{-(n-4)}$ for the Icart mapping. Thanks to AET-DL, choosing $u$ such that the adversary knows both logarithms of $G_{i}$ in basis $f_{i}(u)$ and $G_{j}$ in basis $f_{j}(u)$ is hard. Thus either the map $R_{i} \mapsto \mathrm{CDH}_{f_{i}(u)}\left(G_{i}, R_{i}\right)$ or the map $R_{j} \mapsto \mathrm{CDH}_{f_{j}(u)}\left(G_{j}, R_{j}\right)$ is one way. Consequently, it is computationally hard to find a pair $\left(R_{i}, R_{j}\right)$.

As for the AET-DL assumption, the proof of the validity of the AET-CDH assumption in the generic group model is given in [1]. 
Remark 4. The AET-DL assumption is stronger than the DL assumption. Likewise, AET-CDH is a stronger assumption than the $\mathrm{CDH}$ assumption over any elliptic curve $E_{i}$. Indeed, AET-CDH is trivial if, for one curve $E_{j}, \mathrm{CDH}$ is an easy problem. The following algorithm illustrates this.

1. Randomly select $r_{i}$, compute $P_{i}=r_{i} \cdot G_{i}, R_{i}=r_{i} \cdot F_{i}(l)$.

2. Compute $l^{\prime}=\mathcal{I}_{F_{i}}\left(P_{i}\right)$.

3. Compute $R_{j}=\mathrm{CDH}_{G_{j}}\left(F_{j}(l), F_{j}\left(l^{\prime}\right)\right)$ and return $l^{\prime}, R_{i}, R_{j}$.

We finally introduce a last assumption, which is the password based variant of the AET-DL assumption.

Definition 8 ( $n$-Password Based Admissible Encoding Twin Computational Diffie-Hellman Assumption). Let $P_{\pi}$ be a point over $E_{a_{\pi}, b_{\pi}}$. Let $l$ be an admissible representation of $P_{\pi}\left(P_{\pi}=F_{\pi}(l)\right)$. Let $\mathcal{A}$ be a polynomial algorithm that:

- inputs $S$ and $l$;

- outputs $l^{\prime}, K_{1}, \ldots, K_{n}$, where each $K_{i}$ is a point of one of the curves in $S$.

The n-PAET-CDH assumption holds if any polynomial adversary $\mathcal{A}$ has a probability $1 / N+\varepsilon$ to have returned one $K_{i}$ such that $\mathrm{CDH}_{G_{\pi}}\left(F_{\pi}(l), F_{\pi}\left(l^{\prime}\right)\right)=K_{i}$, where $\varepsilon$ is negligible and $N$ corresponds to the dictionary size (i.e. the number of possible curves).

In this assumption, $\varepsilon$ is the advantage of the algorithm over the value of $\pi$. Indeed, a trivial way to solve the $n$-PAET-CDH problem is, from $S$ and $l$, to randomly choose a $j \in[1, N]$ and to assume that $j=\pi$. This has a probability at least $1 / N$ to succeed. Further, this assumption implies the AET-CDH assumption. Indeed an algorithm $\mathcal{A}^{\text {aet-cdh }}$, which solves the AET-CDH problem, can be transformed into an adversary which solves the $n$-PAET-CDH problem with $\varepsilon=1 / N$. The following lemma proves that the converse is also true.

Lemma 3. The AET-CDH assumption implies the $n$-PAET-CDH assumption, for any $n$.

Proof. Let Succ ${ }^{x}$ be the probability of success of the best adversary against the

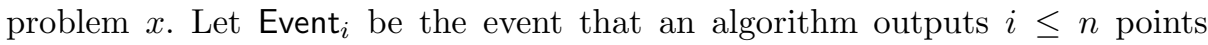
$K_{j_{1}}, \ldots, K_{j_{i}}$ such that

$$
\mathrm{CDH}_{G_{j_{i}}}\left(F_{j_{i}}(l), F_{j_{i}}\left(l^{\prime}\right)\right)=K_{j_{i}}
$$

We have:

$$
\operatorname{Pr}\left[\text { Succ }^{\text {paet-cdh }}\right] \leq \sum_{i=1}^{\min (N, n)} \operatorname{Pr}\left[\text { Event }_{i}\right] \frac{i}{N}
$$

It is easily seen that for all $i \geq 2, \operatorname{Pr}\left[\right.$ Event $\left._{i}\right] \leq \operatorname{Pr}\left[\right.$ Succ $\left.^{\text {aet-cdh }}\right]$. This leads to:

$$
\begin{aligned}
\operatorname{Pr}\left[\text { Succ }^{\text {aet }-c d h}\right] & \leq \operatorname{Pr}\left[\text { Event }_{1}\right] \frac{1}{N}+\operatorname{Pr}\left[\text { Succ }^{\text {aet }-c d h}\right] \frac{N-1}{2} \\
& \leq \frac{1}{N}+\operatorname{Pr}\left[\text { Succ }^{\text {aet }-c d h}\right] \frac{N-1}{2}
\end{aligned}
$$


If $\operatorname{Pr}\left[\right.$ Succ $\left.^{a e t-c d h}\right]$ is negligible, since $N$ is polynomial, then: $\operatorname{Pr}\left[\right.$ Succ $\left.^{\text {paet }-c d h}\right]=$ $\frac{1}{N}+\varepsilon$

\section{The EC-DH-EKE Protocol with an Admissible Encoding}

The Diffie-Hellman Encrypted Key Exchange (DH-EKE) protocol is roughly a DH key exchange where each data sent is encrypted by a block cipher with a key derived from a shared secret. This protocol has been introduced in [5], extended in [3, and proved in the ideal cipher model and random oracle model under the $\mathrm{CDH}$ assumption in 9. Its basic flows are presented in Figure1 (a complete execution, with the final authentication checks, is given in Figure2 in our elliptic curve instantiation).

\begin{tabular}{lrr}
\hline $\begin{array}{l}\text { Device } \\
\text { password } \pi\end{array}$ & parameters : $E, N, G$ & $\begin{array}{r}\text { Reader } \\
\text { password } \pi\end{array}$ \\
\hline $\begin{array}{l}\text { Compute } K_{p w}=H(\pi) \\
\text { Pick } \alpha\end{array}$ & $\begin{array}{r}\text { Compute } K_{p w}=H(\pi) \\
\text { Pick } \beta\end{array}$ \\
Compute $G_{1}=\alpha \cdot G$ & $\frac{z_{1}=\mathcal{E}_{K_{p w}}\left(G_{1}\right)}{z_{2}=\mathcal{E}_{K_{p w}}\left(G_{2}\right)}$ & Compute $G_{2}=\beta \cdot G$ \\
Compute $K=\alpha \cdot \mathcal{D}_{K_{p w}}\left(z_{2}\right)$ & & Compute $K=\beta \cdot \mathcal{D}_{K_{p w}}\left(z_{1}\right)$ \\
\hline
\end{tabular}

Fig. 1. Basic flows of the DH-EKE scheme

Note however that it is assumed that the ideal cipher inputs group elements. Consequently, a naive implementation of the DH-EKE over elliptic curves could be insecure. Indeed, the encryption of a point $P=(x, y)$ with a key $K_{p w}=H(\pi)$ leads to a ciphertext $z=\mathcal{E}_{K_{p w}}(x \| y)$. However, for any password $\pi^{\prime} \neq \pi$, the decryption of $z$ is not a point over the elliptic curve with an overwhelming probability. This leads to an offline dictionary attack (see for instance [8]). More generally, since there exists a redundancy in the representation of $P=(x, y)$, it is difficult to encrypt $P$ without having a dictionary attack. The encryption over the elliptic curves points should in fact be a permutation. One possibility to address this problem is to represent $P$ thanks to an admissible representation. Hence applying a classical cipher would become possible.

\subsection{Parameters}

Let $k$ be a security parameter. Let $\mathcal{H}$ be a hash function with $\{0,1\}^{l}$ as range. Let $N$ be the size of $\mathcal{D}$, the dictionary of the different passwords. Let $E_{a, b}$ be an elliptic curve over $\mathbb{F}_{2^{2 k+1}}$ with an admissible encoding $F$ (and a related inversion algorithm $\mathcal{I}_{F}$ ) and $G$ be a generator of its prime order subgroup of order $q$, with a cofactor 2 .

We assume that the protocol takes place between different devices $D$ and a reader $R$. Each device possesses a password $\pi \in \mathcal{D}$. 


\subsection{EC-DH-EKE}

The DH-EKE scheme has been proved secure in 9 in the ideal cipher model and the random oracle model under the $\mathrm{CDH}$ assumption. However, the ideal cipher requires to manage group elements as inputs.

Thanks to the admissible encoding, a group element can be seen as a bitstring. For this reason, a real implementation of the protocol is much more realistic because the ideal cipher can be instantiated by a cipher such as AES128 in ECB mode, while an ideal cipher over elliptic curve points is still to be found. The resulting protocol is described by Figure 2

\begin{tabular}{|c|c|c|}
\hline Device & & Reader \\
\hline password $\pi$ & parameters : $E_{a, b}, N, G$ & password $\pi$ \\
\hline Compute $K_{p w}=H(\pi)$ & & Compute $K_{p w}=H(\pi)$ \\
\hline Pick $\alpha$ & & Pick $\beta$ \\
\hline Compute $G_{1}=\alpha \cdot G$ & & Compute $G_{2}=\beta \cdot G$ \\
\hline Compute $l_{1}=\mathcal{I}_{F}\left(G_{1}\right)$ & & Compute $l_{2}=\mathcal{I}_{F}\left(G_{2}\right)$ \\
\hline & $z_{1}=\mathcal{E}_{K p w}{ }^{\left(l_{1}\right)}$ & \\
\hline & $z_{2}=\mathcal{E}_{K_{p w}}{ }^{\left(l_{2}\right)}$ & \\
\hline Compute $l_{2}=\mathcal{D}_{K_{p w}}\left(z_{2}\right)$ & & Compute $l_{1}=\mathcal{D}_{K_{p w}}\left(z_{1}\right)$ \\
\hline Compute $K=\alpha \cdot F\left(l_{2}\right)$ & & Compute $K=\beta \cdot F\left(l_{1}\right)$ \\
\hline Compute $\mathcal{K}=\mathcal{H}\left(K, z_{1}, z_{2}\right)$ & & Compute $\mathcal{K}=\mathcal{H}\left(K, z_{1}, z_{2}\right)$ \\
\hline Compute $K_{\text {enc }}=\mathcal{H}(\mathcal{K}, 1)$ & & Compute $K_{\text {enc }}=\mathcal{H}(\mathcal{K}, 1)$ \\
\hline Compute $K_{\mathrm{mac}}=\mathcal{H}(\mathcal{K}, 2)$ & & Compute $K_{\text {mac }}=\mathcal{H}(\mathcal{K}, 2)$ \\
\hline Compute $T_{D}=\mathcal{H}\left(K_{\mathrm{mac}}, z_{2}\right)$ & & Compute $T_{R}=\mathcal{H}\left(K_{\mathrm{mac}}, z_{1}\right)$ \\
\hline & $T_{D}$ & \\
\hline & $T_{R}$ & \\
\hline
\end{tabular}

Fig. 2. The EC-DH-EKE scheme with an Admissible Encoding

This finally leads to an efficient and secure protocol. Additionally, the elliptic curves parameters remain hidden from an eavesdropper, since it only sees some encryption of statistically indistinguishable bit string.

Remark 5. In the masked DH-EKE variant, which is proved in [10] in the ROM only, the encryption primitive is a mask generation function instead of an ideal cipher; the Diffie-Hellman values sent are masked by addition with a full-range hash of the password. Here a similar problem arises: the hash needs to be a ROM into elliptic curves. It is possible to use the [12] ROM construction, which is based on Admissible Encoding, to hash the password. But in that case the elliptic curves parameters will not be kept hidden as the resulting ciphertexts are points on the curve.

Remark 6 (Eavesdroppers without the Elliptic Curve Parameters). The family of DH-EKE protocol is secure against offline dictionary attacks under the CDH assumption: an adversary has to compute $K=\mathrm{CDH}_{G}\left(G_{1}, G_{2}\right)$ to get some information on the password. Indeed, based on $\mathrm{CDH}$ and the ROM, the distribution 
of $G_{1}, G_{2}, T_{D}, T_{R}$ is computationally indistinguishable from the uniform distribution over $E_{a, b}^{2} \times\{0,1\}^{2 l}$. Using this property and the property of the admissible encoding (cf. Definition 2), we know that $l_{1}$ and $l_{2}$ are bit strings computationally indistinguishable from random ones. In the ideal cipher model, this implies that the $z_{1}, z_{2}, T_{D}, T_{R}$ are indistinguishable from random strings as well. For this reason, an adversary who does not know the elliptic curve parameters, cannot compute them, even if he has a list of curves parameters.

\section{Our Proposal of Password Based EC-DH Key Exchange without Encryption}

In the EC-DH-EKE scheme (Figure 2), we use the admissible representation in order to encrypt properly elliptic curves points. As an additional benefit, this protocol also ensures the privacy of the elliptic curve parameters. Following this last idea, we modify further the EC-DH-EKE protocol in order to base the authentication directly on the knowledge of the elliptic curve parameters instead of the knowledge of an additional password.

Our proposal is similar to our EC-DH-EKE variant: points are represented by an admissible representation. But we did not encrypt the representations anymore. Since the distribution of $l_{1}, l_{2}, T_{D}, T_{R}$ is computationally indistinguishable from the uniform distribution, exchanging these values in clear makes no difference from an eavesdropper point of view. This enables to avoid the use of an ideal cipher in the security analysis. In the sequel, we denote our scheme EC-DHARKE which stands for Elliptic Curve Diffie-Hellman Admissibly Represented Key Exchange.

In our scheme, the dictionary of passwords becomes a set of different elliptic curves parameters indexed by a table. This table is not secret. Before an authentication, the device holder typesets an index over the reader, the latter can then verifies whether this index corresponds to the stored elliptic curve parameters.

\subsection{Parameters}

Let $k$ be a security parameter and $N$ a polynomial integer in $k$. Let $\mathcal{H}_{0}, \mathcal{H}_{1}, \mathcal{H}_{2}$ be 3 hash functions with $\{0,1\}^{l}$ as range.

Let $\mathbb{F}_{2^{n}}$ be a field such that there exist efficient admissible encodings and such that $2^{n}=\mathcal{O}\left(2^{2 k}\right)$. Let $S=\left\{a_{i}, b_{i}, G_{i}, q_{i}\right\}_{i \in[1, N]}$ be a set of elliptic curve parameters such that $G_{i}$ is a generator of the prime order group of $E_{i}=E_{a_{i}, b_{i}}$ of order $q_{i}$ and let $F_{i}$ be the associated admissible encoding. It is assumed that the cofactor is the same (2) for each group of points. We also assume that the prime integers $q_{i}$ are pair-wise distinct. This last condition is sufficient to ensure that no isomorphism exists between any pair $\left(E_{i}, E_{j}\right)$ with $i \neq j$.

\subsection{The EC-DH-ARKE Protocol}

During the initialization phase, each reader receives the set $S$ as input and each device receives one element of $S$ as parameters. It can further define its own 
public discrete logarithm based pair of public/secret keys with these parameters. The index $i$ related to these parameters is given to the device owner. We stress that the set $S$ does not need to remain secret. We use the index in order to enable a user to typeset data related to the parameter.

At the beginning of each authentication, the device holder has to typeset one index and then the reader verifies that the index corresponds to the elliptic curve parameters used by the device. The protocol is illustrated in Figure 3 .

\begin{tabular}{|c|c|c|}
\hline Device & & Reader \\
\hline password : $E_{a_{\pi}, b_{\pi}}, q_{\pi}, G_{\pi}$ & & password : $E_{a_{\pi}, b_{\pi}}, q_{\pi}, G_{\pi}$ \\
\hline Pick $\alpha$ & & Pick $\beta$ \\
\hline Compute $G_{1}=\alpha \cdot G_{\pi}$ & & Compute $G_{2}=\beta \cdot G_{\pi}$ \\
\hline \multirow[t]{3}{*}{ Compute $l_{1}=\mathcal{I}_{F_{\pi}}\left(G_{1}\right)$} & & Compute $l_{2}=\mathcal{I}_{F_{\pi}}\left(G_{2}\right)$ \\
\hline & $l_{1}$ & \\
\hline & $l_{2}$ & \\
\hline Compute $\begin{aligned} K & =\alpha \cdot G_{2} \\
& =\alpha \cdot F_{\pi}\left(l_{2}\right)\end{aligned}$ & & $\begin{array}{r}\text { Compute } K=\beta \cdot G_{1} \\
=\beta \cdot F_{\pi}\left(l_{1}\right)\end{array}$ \\
\hline Compute $\mathcal{K}=\mathcal{H}_{0}\left(K, l_{1}, l_{2}\right)$ & & Compute $\mathcal{K}=\mathcal{H}_{0}\left(K, l_{1}, l_{2}\right)$ \\
\hline Compute $K_{\text {enc }}=\mathcal{H}_{1}(\mathcal{K}, 1)$ & & Compute $K_{\text {enc }}=\mathcal{H}_{1}(\mathcal{K}, 1)$ \\
\hline Compute $K_{\text {mac }}=\mathcal{H}_{1}(\mathcal{K}, 2)$ & & Compute $K_{\text {mac }}=\mathcal{H}_{1}(\mathcal{K}, 2)$ \\
\hline \multirow[t]{3}{*}{ Compute $T_{D}=\mathcal{H}_{2}\left(K_{\mathrm{mac}}, l_{2}\right)$} & & Compute $T_{R}=\mathcal{H}_{2}\left(K_{\text {mac }}, l_{1}\right)$ \\
\hline & $T_{D}$ & \\
\hline & $T_{R}$ & \\
\hline Abort if $T_{R}$ invalid & & Abort if $T_{D}$ invalid \\
\hline
\end{tabular}

Fig. 3. Our proposal EC-DH-ARKE

\subsection{Security Result}

Our proposal is secure in the random oracle model under the AET-CDH assumption. More concretely:

Theorem 1 (AKE security). Let $S$ be a randomly chosen set of $N$ elliptic curve parameters as above. Let $\pi$ be an uniformly chosen index in $[1, N]$. Assume that $\mathcal{H}_{0}, \mathcal{H}_{1}, \mathcal{H}_{2}$ are random oracles. Let $\mathcal{A}$ be an adversary in the BPR model against the AKE security of our scheme within a time $T$, with less than $q_{s}$ interactions with the parties, $q_{p}$ eavesdroppings and $q_{h}$ hash queries. We have:

$$
\operatorname{Adv}_{\mathrm{EC}-\mathrm{DH}-\operatorname{ARKE}}^{\text {ake }}(\mathcal{A}) \leq q_{s} \operatorname{Succ}^{q_{h}-\text { paet-cdh }}\left(T^{\prime}\right)+q_{p} \operatorname{Succ}^{\mathrm{cdh}}\left(T^{\prime}\right)+\varepsilon
$$

where $T^{\prime}=T+\mathcal{O}\left(Q^{2}\right)$, where $Q=q_{s}+q_{h}+q_{p}$ and $\varepsilon$ is negligible if $q_{s}, q_{p}$ and $q_{h}$ are polynomial in $k$.

The security of this protocol relies on two ideas:

1. a passive eavesdropper does not get any information on the exchanged data whenever the CDH is a hard problem for any curve in $S$;

2. an active adversary can find the password by an online dictionary attack with a probability $1 / N$. In fact, an adversary can always be turned into an algorithm, which solves the PAET-CDH problem with almost the same probability. 
Remark 7. By definition of an admissible encoding, the inversion algorithm runs in probabilistic time (see Definition 2), thus the implementation of the protocol could result on a non-constant execution runtime (that is depending of the curve used, i.e. of the password); which in a practical application may represent a privacy risk. In [11], we explain that a polynomial adversary cannot exploit this information to distinguish two curves.

\subsection{Security Proof}

Proof. We use a sequence of game in order to prove the security of the protocol. In the sequel $\operatorname{Pr}\left[G_{i}\right]$ denotes the probability in the game $G_{i}$ that the adversary outputs the good guess $b^{\prime}=b$.

Game $G_{0}$ : This is the real security game. A set of $N$ parameters is chosen, the device receives one element of $S$ and the reader receives the same, while the set $S$ is given to the adversary. The reader and the device act as described in Figure 3. We assume that $\mathcal{H}_{0}, \mathcal{H}_{1}, \mathcal{H}_{2}$ are random oracles into $\{0,1\}^{l}$.

Once the TEST query is sent, following a randomly chosen bit $b$, the key $K_{\text {enc }}$ or a random string is returned. Hence

$$
\operatorname{Adv}_{\mathrm{EC}-\mathrm{DH}-\mathrm{ARKE}}^{\text {ake }}(\mathcal{A})=\left|\operatorname{Pr}\left[G_{0}\right]-1 / 2\right|
$$

Game $G_{1}$ : We simulate the device and the reader for each query to the SEND, Execute, Test and Reveal oracles, as the real players would do. We also simulate the random oracles $\mathcal{H}_{0}, \mathcal{H}_{1}, \mathcal{H}_{2}$. This does not change the adversary advantage but modifies the duration of the simulation because of the necessary table lookups. We thus have $T^{\prime}\left[G_{1}\right]=T+\mathcal{O}\left(Q^{2}\right)$.

Game $G_{2}$ : We abort the simulation if a collision occurs while simulating one of the random oracles. A collision occurs with a probability $Q^{2} / 2^{l+1}$ for each random oracle. We thus have:

$$
\left|\operatorname{Pr}\left[G_{2}\right]-\operatorname{Pr}\left[G_{1}\right]\right| \leq 5 \times \frac{Q^{2}}{2^{l+1}}
$$

From this game, we are almost sure that the values of $\mathcal{K}$ are different. This property is also true for $K_{\text {enc }}, K_{\text {mac }}, T_{D}, T_{R}$.

Game $G_{3}$ : We simulate the ExECUTE oracle using random values. To distinguish this game from the previous one, the adversary needs to solve the $\mathrm{CDH}$ problem over a curve for at least one pair $\left(l_{1}, l_{2}\right)$ exchanged during one of the ExECUTE queries. For this reason, we have:

$$
\left|\operatorname{Pr}\left[G_{3}\right]-\operatorname{Pr}\left[G_{2}\right]\right| \leq q_{p} \operatorname{Succ}^{c d h}\left(T^{\prime}\right)
$$

Game $G_{4}$ : We abort the simulation when we get a collision on elliptic curve points chosen at the beginning. Since there are $q_{\pi}$ points in the curve, we have that:

$$
\left|\operatorname{Pr}\left[G_{4}\right]-\operatorname{Pr}\left[G_{3}\right]\right| \leq \frac{Q^{2}}{q_{\pi}}
$$


From this game, we know that the admissible representations returned by the simulation are pair-wise distinct.

Game $G_{5}$ : We abort when one triplet $\left(\mathrm{CDH}_{G_{\pi}}\left(F_{\pi}\left(l_{1}\right), F_{\pi}\left(l_{2}\right)\right), l_{1}, l_{2}\right)$ is queried a second time to $\mathcal{H}_{0}$, while $l_{1}, l_{2}$ are values exchanged during one instance $I$ initiated by the query SEND. When this second query to $\mathcal{H}_{0}$ occurs, we know that the adversary knows the value $\mathcal{K}$ of the instance $I$. We have assumed that the adversary does not make two identical queries to any random oracle.

Before this abortion, the adversary does not have any advantage over the password $\pi$ since he has observed random values (due to the admissible representations property, see Section 2.1) that he could not verify, without querying $\mathcal{H}_{0}$ with a correct query. Once this event happens, we determine $l$, the value amongst $l_{1}$ or $l_{2}$, that we sent when we simulated the SEND query. We then get all the triplets queried to the random oracle $\mathcal{H}_{0}$ by the adversary, which contains $l$. These triplets form an answer to the PAET-CDH problem. For this reason we have:

$$
\left|\operatorname{Pr}\left[G_{5}\right]-\operatorname{Pr}\left[G_{4}\right]\right| \leq q_{s} \text { Succ }^{q_{h}-\text { paet }- \text { cdh }}\left(T^{\prime}\right)
$$

Game $G_{6}$ : We do not use $\mathcal{H}_{1}$ and $\mathcal{H}_{2}$ anymore when we simulate the execution of the protocol. For this reason, the REVEAL query does not give any information such as the SEND query concerning $T_{R}$ and $T_{D}$. We thus have:

$$
\operatorname{Pr}\left[G_{6}\right]=1 / 2
$$

Furthermore, since the adversary did not compute $\mathcal{K}$ in any instance, there is no difference from the adversary point of view between $G_{5}$ and $G_{6}$. So $\operatorname{Pr}\left[G_{5}\right]=$ $\operatorname{Pr}\left[G_{6}\right]$.

Note that in the above security result, the ROM hypothesis is needed only to simulate the flow part $\left(T_{D}, T_{R}\right)$, the ROM is not used in the simulation of the first part (key exchange, $l_{1}, l_{2}$ ) of the protocol. Only our complexity assumptions (cf. Section 3) are necessary for this part. This result holds in the forward secrecy setting (cf. Remark 1) as well.

\section{Conclusion and Further Works}

This paper describes a new and efficient Password-based Authenticated Key Exchange protocol which is especially adapted for elliptic curves. Particularly, it enables to keep the elliptic curves parameters hidden. In the context of contactless ID documents, this opens a way for implementing realistic solutions which preserve privacy of the owners; especially their nationality. As extensions of this work, a good perspective is to apply our technique on the enhanced versions of EKE which are secure in a more general model (e.g. UC) or with standard assumptions (standard model).

An implementation in a PKI context with the property that the curve parameters remain hidden is also a possible application of our idea. For instance, 
a smartcard could contain a certified public key which is stored directly in its admissible representation.

Note that the implementation issues are discussed in the characteristic 2 context for simplicity. Applications to characteristic $p>2$ when $p$ is approximately a power of 2 (i.e. a pseudo-Mersenne prime as for the curves recommended by NIST) are also possible.

Acknowledgements. The authors thank the reviewers for their comments.

\section{References}

1. Abdalla, M., Fouque, P.-A., Pointcheval, D.: Password-based authenticated key exchange in the three-party setting. In: Vaudenay, S. (ed.) PKC 2005. LNCS, vol. 3386, pp. 65-84. Springer, Heidelberg (2005)

2. Barreto, P.: Why public elliptic curves parameters are public, Tales from the Cryptographers (2005), http://www.larc.usp.br/ pbarreto/tales1.html

3. Bellare, M., Pointcheval, D., Rogaway, P.: Authenticated key exchange secure against dictionary attacks. In: Preneel, B. (ed.) EUROCRYPT 2000. LNCS, vol. 1807, pp. 139-155. Springer, Heidelberg (2000)

4. Bellare, M., Rogaway, P.: The AuthA protocol for password-based authenticated key exchange. In: IEEE P1363, pp. 136-3 (2000)

5. Bellovin, S.M., Merritt, M.: Encrypted key exchange: Password-based protocols secure against dictionary attacks. In: IEEE Symposium on Research in Security and Privacy, pp. 72-84 (1992)

6. Bellovin, S.M., Merritt, M.: Augmented encrypted key exchange: A password-based protocol secure against dictionary attacks and password file compromise. In: ACM Conference on Computer and Communications Security, pp. 244-250 (1993)

7. Boneh, D., Franklin, M.K.: Identity-based encryption from the weil pairing. In: Kilian, J. (ed.) CRYPTO 2001. LNCS, vol. 2139, pp. 213-229. Springer, Heidelberg (2001)

8. Boyd, C., Montague, P., Nguyen, K.Q.: Elliptic curve based password authenticated key exchange protocols. In: Varadharajan, V., Mu, Y. (eds.) ACISP 2001. LNCS, vol. 2119, pp. 487-501. Springer, Heidelberg (2001)

9. Bresson, E., Chevassut, O., Pointcheval, D.: Security proofs for an efficient password-based key exchange. In: Jajodia, S., Atluri, V., Jaeger, T. (eds.) ACM Conference on Computer and Communications Security, pp. 241-250. ACM, New York (2003)

10. Bresson, E., Chevassut, O., Pointcheval, D.: New security results on encrypted key exchange. In: Bao, F., Deng, R., Zhou, J. (eds.) PKC 2004. LNCS, vol. 2947, pp. 145-158. Springer, Heidelberg (2004)

11. Bringer, J., Chabanne, H., Icart, T.: Password based key exchange with hidden elliptic curve public parameters. Cryptology ePrint Archive, Report 2009/468 (2009), http://eprint.iacr.org/

12. Coron, J.-S., Icart, T.: A random oracle into elliptic curves. Cryptology ePrint Archive, Report 2009/340 (2009), http://eprint.iacr.org/

13. Icart, T.: How to hash into elliptic curves. In: Halevi, S. (ed.) CRYPTO 2009. LNCS, vol. 5677, pp. 303-316. Springer, Heidelberg (2009) 
14. Jablon, D.P.: Strong password-only authenticated key exchange. ACM Computer Communications Review 26, 5-26 (1996)

15. International Civil Aviation Organization. Machine readable travel documents website, http:/mrtd.icao.int/

16. Patel, S.: Number theoretic attacks on secure password schemes. In: IEEE Symposium on Security and Privacy, pp. 236-247. IEEE Computer Society, Los Alamitos (1997)

17. Shallue, A., van de Woestijne, C.: Construction of rational points on elliptic curves over finite fields. In: Hess, F., Pauli, S., Pohst, M.E. (eds.) ANTS 2006. LNCS, vol. 4076, pp. 510-524. Springer, Heidelberg (2006)

18. Ullmann, M., Kugler, D., Neumann, H., Stappert, S., Vogeler, M.: Password authenticated key agreement for contactless smart cards. In: RFIDSec (2008) 